\title{
Sexual Function and Breast-Specific Sensuality Remain Important After Breast Cancer Surgery
}

\author{
Sarah A. McLaughlin, MD', Lauren F. Cornell, $\mathrm{MD}^{2}$, and Dawn M. Mussallem ${ }^{2}$ \\ ${ }^{1}$ Department of Surgery, Mayo Clinic Florida, Jacksonville, FL; ${ }^{2}$ Division of Hematology and Oncology, Mayo Clinic \\ Florida, Jacksonville, FL
}

Thanks to improved screening and targeted therapeutics, the survival rates for breast cancer have steadily improved during the last three decades. As a result, breast cancer survivors account for more than 3 million of the 14.5 million cancer survivors currently living in the United States. ${ }^{1}$

The National Cancer Institute expects the numbers of breast cancer survivors to rise to more than 4 million by $2024 .^{2}$ This growing number of survivors has helped to focus clinical decision making on issues beyond active treatment and to encompass discussions on the challenges faced by survivors and on the critical importance of regaining meaningful quality of life. National efforts to improve patient education on lymphedema, habits of healthy living with diet and exercise, risks of obesity, and the survivorship platforms of the American Society of Clinical Oncology (ASCO), National Comprehensive Cancer Network (NCCN), American College of Surgeons' Commission on Cancer (CoC), and the National Accreditation Program for Breast Centers (NAPBC) seek to educate both patients and clinicians with information to bridge these gaps into survivorship. Understanding the needs of the patient during this transition and providing important breast cancer resources on topics including

This is an editorial for the article "Breast specific sensuality and sexual function in cancer survivorship: Does surgical modality matter?" by Jennifer Gass et al. available at doi:10.1245/s10434-0175905-4.

(C) Society of Surgical Oncology 2017

First Received: 2 July 2017;

Published Online: 13 September 2017

S. A. McLaughlin, MD

e-mail: Mclaughlin.sarah@mayo.edu exercise, nutrition, obesity, alcohol avoidance, late effects of treatment such as lymphedema, fear of recurrence, sexuality and fertility, and returning to the workplace are essential.

Yet, despite the intense national survivorship efforts and the growing body of literature recognizing that $50-90 \%$ of breast cancer survivors experience sexual dysfunction after treatment, ${ }^{3,4}$ adequate sexual histories are discussed by less than $50 \%$ of physicians, and probably very few of these are surgeons. ${ }^{3}$ Talking about sexual (dys)function can be equally embarrassing for patient and provider. The provider, who probably has limited time, and the patient may feel as though the discussion is futile or without solution.

The overall failure to address sexual health frequently leads to voids in medical knowledge because baseline sexual health data are unknown, which further limits our ability to quantify post-treatment sexual function change. These baseline data are critical for breast cancer patients because the median age at diagnosis is 62 years, ${ }^{5}$ and many postmenopausal patients may already be experiencing some type of sexual decline at the time of diagnosis. ${ }^{6}$ This recent article by Gass et al. should encourage surgeons to reconsider their willingness to discuss with their patients the sexual side effects resulting from surgery and their approach to the discussion.

In the accompanying report, Gass et al. argues that the breast is an integral sensual organ and reports on the novel correlation between breast-specific sensuality (BSS) as assessed by a 7-item questionnaire focused on the breast in intimacy and pleasure and sexual function as assessed by the Female Sexual Function Index (FSFI), a 19-item validated tool assessing desire, arousal, lubrication, orgasm, satisfaction, and pain.

After stratifying for surgical procedure, the investigators found that patient importance of the chest to intimacy and sex declined after surgery in all groups (all $p<0.003$ ) but 
noted that it remained important for a substantial number of women (74\% of lumpectomy patients; $77 \%$ of mastectomy and reconstruction patients). However, significantly more lumpectomy patients reported breast appearance satisfaction and pleasurable breast caress $(p=0.02)$ than patients who underwent mastectomy with reconstruction $(p=0.01)$.

The FSFI scores did not differ among surgical procedures. However, when the FSFI scores were re-stratified according to BSS questions, those with higher BSS satisfaction not only had higher median FSFI scores, but also had median scores (all $>28.2$; Fig. 2) well above the validated threshold for sexual dysfunction (FSFI score, $<26.55$ ). Extrapolation of these data collectively suggests that women having lumpectomy are ultimately more satisfied and have higher sexual function than those having mastectomy with or without reconstruction.

Based on the clear importance of the breast as a sensual organ and these data by Gass et al., more cosmetic mastectomies (i.e., skin-sparing or nipple-sparing), although oncologically safe and cosmetically superior, may not necessarily resolve the sexual dysfunction changes seen in breast cancer patients. Certainly, this needs to be addressed as the body of literature on nipple-sparing mastectomy increases.

Unfortunately, but clearly acknowledged by the authors in the discussion, their analysis was limited to the surgical treatment and could not further stratify BSS outcomes by receipt of adjuvant radiotherapy, chemotherapy, or endocrine therapy. Table 1 highlights the differences in radiation and chemotherapy (both $p<0.01$ ) and the similarities in the receipt of endocrine therapy ( $p=1.0)$ among the groups. Although it is assumed that these can influence BSS, the authors cite this as an area of ongoing research.

For us providers, it is hard to discuss sex with our patients. Recent data, however, suggest that we should.
Most domains of sexual function decline after breast cancer treatment except for the patient's desire for sex. ${ }^{6}$ Recognizing that women desire sex after treatment, we should try to protect and preserve sexual function. Gass et al. showed that surgery affects function. We should inform our patients about the sexual side effects of procedures just as we advise them on the risk of hematoma, infection, lymphedema, and recurrence, and on the decades of data supporting the oncologic equivalence of breast conservation and mastectomy, and just as we provide patientcentered guidance in the surgical decision-making process.

Finally if we believe the old advertising adage that "sex sells," then perhaps a robust discussion on the influence of the surgical procedure on sexual function may help to convince eligible breast-conservation patients to choose breast conservation. Perhaps sex can help us sway the mastectomy trend in the United States in early-stage breast cancer, where traditional surgical facts and data on survival and complications have not.

\section{REFERENCES}

1. DeSantis CE, Lin CC, Mariotto AB, et al. Cancer treatment and survivorship statistics, 2014. CA Cancer J Clin. 2014;64:252-71.

2. Surveillance, Epidemiology and End Results Program. https://seer. cancer.gov/statfacts/html/breast.html.

3. Dizon DS. Quality of life after breast cancer: survivorship and sexuality. Breast J. 2009;15:500-4.

4. Ussher JM, Perz J, Gilbert E, et al. Talking about sex after cancer: a discourse analytic study of health care professional accounts of sexual communication with patients. Psychol Health 2013;28:1370-90.

5. Komen SG. 2017. https://ww5.komen.org/BreastCancer/ GettingOlder.html. Accessed 1 July 2017.

6. Cornell LF, Mussallem DM, Gibson TC, et al. Trends in sexual function after breast cancer surgery. Ann Surg Oncol. 2017. doi:10. 1245/s10434-017-5894-3. 